\title{
Editorial
}

\section{Advances in Boundary-Layer/Air Pollution Meteorology}

\author{
Xiao-Ming Hu, ${ }^{1,2}$ Jianping Huang, ${ }^{3,4}$ Jose D. Fuentes, ${ }^{5}$ Renate Forkel, ${ }^{6}$ and Ning Zhang ${ }^{7}$ \\ ${ }^{1}$ Nanjing University of Information Science and Technology, Nanjing 210044, China \\ ${ }^{2}$ Center for Analysis and Prediction of Storms and School of Meteorology, University of Oklahoma, Norman, OK 73072, USA \\ ${ }^{3}$ Yale-NUIST Center on Atmospheric Environment, Nanjing University of Information Science and Technology, Nanjing 210044, China \\ ${ }^{4}$ I.M. System Group, NOAA/NCEP/Environmental Modeling Center, College Park, MD 20740, USA \\ ${ }^{5}$ Department of Meteorology and Atmospheric Science, Pennsylvania State University, University Park, PA 16802, USA \\ ${ }^{6}$ Karlsruher Institut für Technologie (KIT), IMK-IFU, 82467 Garmisch-Partenkirchen, Germany \\ ${ }^{7}$ Nanjing University, Nanjing 210093, China
}

Correspondence should be addressed to Xiao-Ming Hu; xhu@ou.edu

Received 26 May 2016; Accepted 26 May 2016

Copyright (C) 2016 Xiao-Ming Hu et al. This is an open access article distributed under the Creative Commons Attribution License, which permits unrestricted use, distribution, and reproduction in any medium, provided the original work is properly cited.

Air pollution plagues public health in many areas around the world. Once emitted, the concentrations of ambient air pollutants are determined by a combination of chemical and dispersion processes in the atmospheric boundary layer. The thermodynamic and dynamic state of the atmospheric boundary layer influence these processes in different ways, thereby playing an important role in modulating the ambient air quality. Better understanding of boundary-layer meteorology is warranted for us to reduce air pollution around the world. This special issue includes selected articles that report recent investigations/advances in Boundary-Layer/Air Pollution Meteorology through observational and numerical approaches.

Land-surface processes dictate the evolution of the atmospheric boundary layer by providing forcing from the lower boundary. A double-deck surface air layer model was proposed recently to estimate the evaporation from a running water surface. This model is evaluated/validated in the paper by S. Liu et al. using the field observations conducted over irrigation canals in Heihe River Basin in Northwestern China.

The Community Land Model (CLM) is one of the most widely used models for the description of land-surface processes. The calculation of the hydraulic properties of frozen soil in CLM was recently improved to alleviate the soil dry bias. As shown by X. Fang et al., the updated landsurface model results only in a slightly more realistic climate prediction.
Advanced techniques such as Lidar are often used to monitor the atmospheric boundary layer. Lidar backscatter signals were processed by R. Dang et al. to diagnose the depth of the atmospheric boundary layer, thus monitoring its temporal evolution and its correlation to thermal parameters over a semiarid region in China.

Routine monitoring of near-surface pollutants has been started in China since around 2000. L. Huang et al. analyzed air quality data together with meteorological data in order to investigate the air quality trend in the Anhui province.

Advanced numerical models were developed to simulate boundary-layer meteorology. A nonhydrostatic atmospheric model using the multimoment constrained finite volume scheme was developed by X. Li et al., which was shown to provide better simulations of atmospheric flows over complex terrains. Such numerical techniques can potentially be used to improve air quality simulation over complex mountainous terrain.

Numerical simulations permit investigating the interrelation between boundary-layer processes and air pollution events. X. Wei et al. examined the formation mechanism of photochemical smog associated with the passage of Typhoon Haitang using a mesoscale meteorological model and the Community Multiscale Air Quality (CMAQ) model. They could identify three meteorological factors which were contributing to the photochemical smog. 
Planetary boundary-layer (PBL) parameterizations are critical to air quality modeling. It is not well quantified how air quality simulations are constrained by PBL parameterization schemes under the heavy haze polluted boundary-layer conditions. $\mathrm{T}$. Li et al. present a comprehensive evaluation study to address the importance of PBL parameterization schemes in WRF/Chem predictions of particles with diameters less than 2.5 microns $\left(\mathrm{PM}_{2.5}\right)$ during February 2014 over the most polluted regions in China where $\mathrm{PM}_{2.5}$ concentrations higher than $500 \mu \mathrm{g} \cdot \mathrm{m}^{-3}$ were observed.

Regional transport and local emissions are the two major sources modulating regional air quality. Accurate quantification of air pollutant sources may provide scientific evidence for the development of effective emission control measures. J. Mai et al. performed a series of numerical sensitivity studies to determine the contributions of regional transport and local sources to $\mathrm{PM}_{2.5}$ concentrations in Zhongshan, one of the major cities in the Pearl River Delta region. The authors point out that the relative contributions of pollution sources are highly associated with synoptic weather patterns.

Meteorological services can provide timely information to inform the public on the temporal and spatial distribution of pollutants. In the past decade, such meteorological services in China were rapidly growing to help mitigate the damage of meteorological and air quality hazards, which have increasingly become sources of concern in China in recent years. Z. Kang et al. review the current status of environmental meteorological services provided by the China Meteorological Administration, which include monitoring, forecasting, and early warning of hazardous events such as fog, haze, and dust.

The compiled papers provide timely research results to the readers, thereby gauging the recent advances in Boundary-Layer/Air Pollution Meteorology in China where air quality has become an environmental concern.

Xiao-Ming $\mathrm{Hu}$ Jianping Huang Jose D. Fuentes

Renate Forkel Ning Zhang 

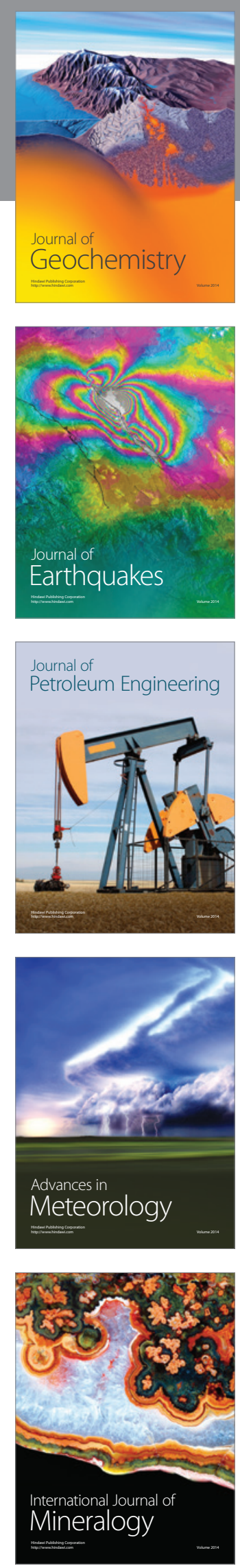
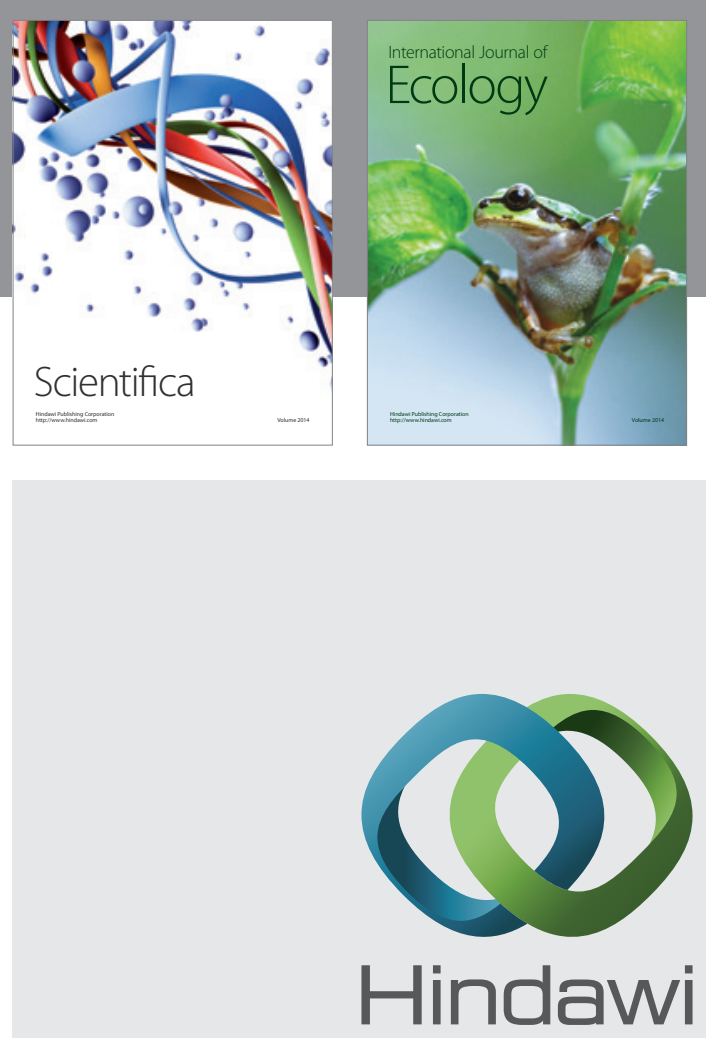

Submit your manuscripts at

http://www.hindawi.com
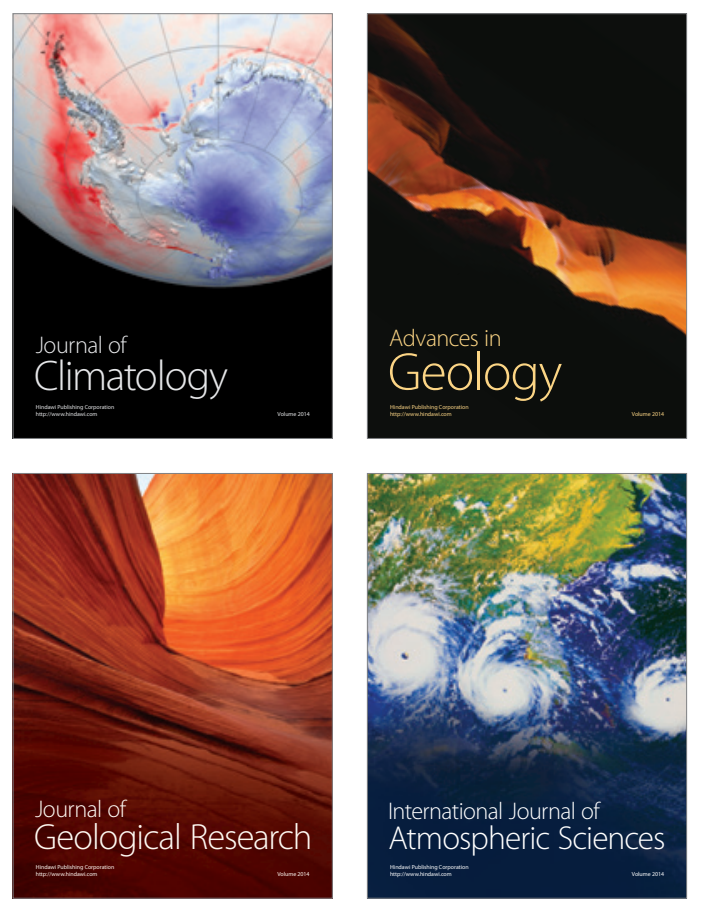

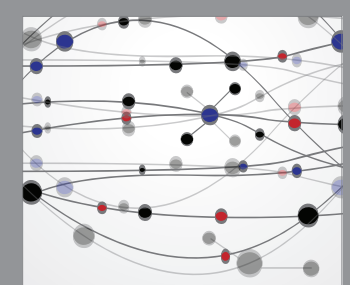

The Scientific

\section{World Journal}
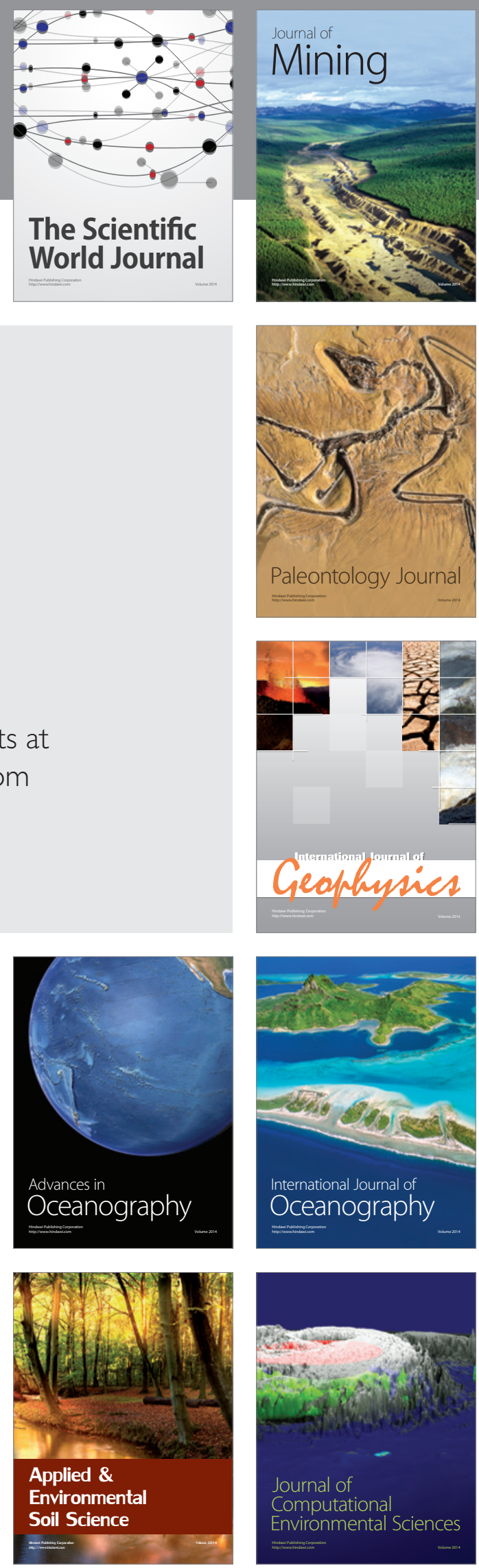